\title{
Stability of generalized Cauchy equations
}

\author{
Roman Badora, Barbara Przebieracz and Peter Volkmann \\ Dedicated to Professor János Aczél on his 90th Birthday
}

Abstract. We investigate the stability of the functional equation

$$
f(x y)=g(x) h(y)+k(y)
$$

on amenable semigroups. This equation is a common generalization of two Pexider equations stemming from Cauchy's additive and multiplicative functional equations, and it is a simple case of the Levi-Civita equation.

Mathematics Subject Classification. Primary 39B82; Secondary 39B22.

Keywords. Cauchy equations, stability in the sense of Hyers-Ulam, Pexider equations, LeviCivita functional equation.

\section{Introduction}

A common generalization of Pexider's equations

$$
f(x y)=g(x)+k(y)
$$

and

$$
f(x y)=g(x) h(y)
$$

is

$$
f(x y)=g(x) h(y)+k(y) .
$$

For real functions this equation has already been treated in J. Aczél's fundamental monograph [1], where the composition $x y$ means addition of real numbers (therefore we have $f(x+y)=g(x) h(y)+k(y)$ on p. 120 of [1]). Then chapter 15 of the book [3] is devoted to equation (1.1). By referring to J. Aczél [2], it is solved under the assumptions that $f, g, h, k: S \rightarrow \mathbb{F}$, where $S$ is an abelian groupoid with neutral element and $\mathbb{F}$ is a field. More precisely, when solving Exercise 1 on p. 250 of [3], it turns out that $f, g, h, k$ satisfy (1.1) if and only if they have one of the following forms (where $b, c, u, v \in \mathbb{F}$ ): 
1. $f(x)=b, g$ arbitrary, $h=0, k(x)=b$.

2. $f(x)=b, g(x)=c, h$ arbitrary, $k(x)=b-c h(x)$.

3. $f(x)=v(a(x)+b), g(x)=a(x)+b-c, h(x)=v, k(x)=v(a(x)+c)$, where $a: S \rightarrow \mathbb{F}$ solves the additive Cauchy equation

$$
a(x y)=a(x)+a(y), \quad x, y \in S .
$$

4. $f(x)=v(c e(x)+b), g(x)=c e(x)+u, h(x)=v e(x), k(x)=v(b-u e(x))$, where $e: S \rightarrow \mathbb{F}$ solves the multiplicative Cauchy equation

$$
e(x y)=e(x) e(y), \quad x, y \in S .
$$

The just given four types of functions $f, g, h, k: S \rightarrow \mathbb{F}$ are also solutions of (1.1) in arbitrary (not necessarily commutative) groupoids $S$.

Equation (1.1) is a special case of the Levi-Civita functional equation

$$
f(x y)=\sum_{i=1}^{n} g_{i}(x) h_{i}(y) .
$$

For information concerning the solutions of this equation see, for example, [5$10]$ and [12]. Recently, solutions of (1.1), on various non-abelian groups, have been examined in [4].

In the present paper we investigate the stability in the sense of Hyers-Ulam of equation (1.1) on amenable semigroups. Let us recall that a semigroup $S$ is called right amenable if there exists a right invariant mean on the space $\mathcal{B}(S)$ of all bounded complex-valued functions defined on $S$. By a right invariant mean we understand a linear functional $M$ satisfying

$$
\begin{gathered}
M(\bar{f})=\overline{M(f)}, \quad f \in \mathcal{B}(S), \\
\inf _{s \in S} f(s) \leq M(f) \leq \sup _{s \in S} f(s), \text { for all real-valued } f \in \mathcal{B}(S),
\end{gathered}
$$

and

$$
M\left(f_{x}\right)=M(f), \quad f \in \mathcal{B}(S), x \in S,
$$

where $f_{x}(s):=f(s x), s \in S$. It is easily seen that the linear functional $M$ has the properties

$$
\|M\| \leq 2
$$

and $M(c)=c$, for all complex numbers $c$. Moreover, for convenience, we will write $M_{s}(f(s))$ instead of $M(f)$.

In paper [11] the stability of the functional equation

$$
u(L x)=\alpha(L) u(x)+\beta(L)
$$

is under consideration, where $X$ is a set, $\mathcal{L}$ is an amenable group of selfmappings of $X, \mathbb{K}$ is the field of real or complex numbers, $u: X \rightarrow \mathbb{K}, \alpha, \beta: \mathcal{L} \rightarrow$ $\mathbb{K}$.

The main result of our paper is contained in the next section. 


\section{Main theorem}

Theorem 2.1. Let $S$ be a right amenable semigroup with neutral element 1 . Suppose $f, g, h, k: S \rightarrow \mathbb{C}, \varepsilon \geq 0$ and

$$
|f(x y)-g(x) h(y)-k(y)| \leq \varepsilon, \quad x, y \in S .
$$

Then there exist $F, G, H, K: S \rightarrow \mathbb{C}$ satisfying

$$
F(x y)=G(x) H(y)+K(y), \quad x, y \in S,
$$

so that the differences $f-F, g-G, h-H$ and $k-K$ are bounded.

We start with two lemmas.

Lemma 2.2. Suppose that $S$ is a semigroup, $\varphi, \psi, \xi: S \rightarrow \mathbb{C}, \delta \geq 0$, $\varphi$ is unbounded and

$$
|\varphi(x y)-\varphi(x) \psi(y)-\xi(y)| \leq \delta, \quad x, y \in S .
$$

Then

$$
\psi(x y)=\psi(x) \psi(y), \quad x, y \in S .
$$

Proof. Let $\left(z_{n}\right)_{n \in \mathbb{N}}$ be a sequence such that

$$
0 \neq\left|\varphi\left(z_{n}\right)\right| \stackrel{n \rightarrow \infty}{\longrightarrow} \infty .
$$

Using (2.3) with $x=z_{n}$ and dividing the obtained inequality by $\left|\varphi\left(z_{n}\right)\right|$, side by side, we have

$$
\left|\frac{\varphi\left(z_{n} y\right)}{\varphi\left(z_{n}\right)}-\psi(y)-\frac{\xi(y)}{\varphi\left(z_{n}\right)}\right| \leq \frac{\delta}{\left|\varphi\left(z_{n}\right)\right|}, \quad n \in \mathbb{N}, y \in S .
$$

Letting $n \rightarrow \infty$ we obtain

$$
\psi(y)=\lim _{n \rightarrow \infty} \frac{\varphi\left(z_{n} y\right)}{\varphi\left(z_{n}\right)}, \quad y \in S .
$$

Using (2.3) with $z_{n} x$ instead of $x$ and dividing the obtained inequality by $\left|\varphi\left(z_{n}\right)\right|$, side by side, we have

$$
\left|\frac{\varphi\left(z_{n} x y\right)}{\varphi\left(z_{n}\right)}-\frac{\varphi\left(z_{n} x\right)}{\varphi\left(z_{n}\right)} \psi(y)-\frac{\xi(y)}{\varphi\left(z_{n}\right)}\right| \leq \frac{\delta}{\left|\varphi\left(z_{n}\right)\right|}, \quad n \in \mathbb{N}, x, y \in S .
$$

Passing with $n$ to infinity and taking (2.4) into account we infer that

$$
|\psi(x y)-\psi(x) \psi(y)-0| \leq 0, \quad x, y \in S,
$$

which completes the proof. 
Lemma 2.3. Suppose that $S$ is a right amenable semigroup, $\varphi, \psi, \xi: S \rightarrow \mathbb{C}$ and $\delta \geq 0$. If

$$
|\varphi(x y)-\varphi(x) \psi(y)-\xi(y)| \leq \delta, \quad x, y \in S,
$$

and

$$
\psi(x y)=\psi(x) \psi(y), \quad x, y \in S,
$$

then there exists a function $\eta: S \rightarrow \mathbb{C}$ such that

$$
\eta(x y)=\eta(x) \psi(y)+\eta(y), \quad x, y \in S,
$$

and

$$
|\eta(x)-\xi(x)| \leq 2 \delta, \quad x \in S .
$$

Proof. Let $M$ be a right invariant mean on $\mathcal{B}(S)$. By $(2.5)$ we infer that, for every $y \in S$, the mapping

$$
S \ni x \mapsto \varphi(x y)-\varphi(x) \psi(y)
$$

is bounded. Hence we can define $\eta: S \rightarrow \mathbb{C}$ by the formula

$$
\eta(y):=M_{z}(\varphi(z y)-\varphi(z) \psi(y)), \quad y \in S .
$$

For $x, y \in S$ we have

$$
\begin{aligned}
\eta(x y) & =M_{z}(\varphi(z x y)-\varphi(z) \psi(x y)) \\
& =M_{z}(\varphi(z x y)-\varphi(z x) \psi(y)+\varphi(z x) \psi(y)-\varphi(z) \psi(x y)) \\
& =M_{z}(\varphi(z x y)-\varphi(z x) \psi(y)+(\varphi(z x)-\varphi(z) \psi(x)) \psi(y)) \\
& =M_{z}(\varphi(z x y)-\varphi(z x) \psi(y))+M_{z}(\varphi(z x)-\varphi(z) \psi(x)) \psi(y) \\
& =\eta(y)+\eta(x) \psi(y) .
\end{aligned}
$$

Moreover, since $\|M\| \leq 2$ (cf.(1.2)), for $y \in S$ we have

$$
\begin{aligned}
|\eta(y)-\xi(y)| & =\left|M_{z}(\varphi(z y)-\varphi(z) \psi(y))-\xi(y)\right| \\
& =\left|M_{z}(\varphi(z y)-\varphi(z) \psi(y))-M_{z}(\xi(y))\right| \\
& =\left|M_{z}(\varphi(z y)-\varphi(z) \psi(y)-\xi(y))\right| \\
& \leq 2 \sup _{z \in S}|\varphi(z y)-\varphi(z) \psi(y)-\xi(y)|,
\end{aligned}
$$

which jointly with (2.5) gives (2.6) and completes the proof.

Proof of Theorem 2.1. From (2.1) we get

$$
|f(x)-g(x) h(1)-k(1)| \leq \varepsilon, \quad x \in S .
$$

By (2.7), applied for $x y$, and (2.1) we obtain

$$
|g(x y) h(1)+k(1)-g(x) h(y)-k(y)| \leq 2 \varepsilon, \quad x, y \in S .
$$

Let us consider the following cases. 
1. $h=0$.

Then, by (2.8), $k$ is bounded, and by (2.7), so is $f$. Functions $F=0$, $G=g, H=0$ and $K=0$ are as required.

2. $h(1)=0$ and there is an $x_{0} \in S$ with $h\left(x_{0}\right) \neq 0$.

By (2.8) with $y=x_{0}$ we see that $g$ is bounded, and therefore, by (2.7), $f$ is also bounded.

- If $g(x)=a \in \mathbb{C}$ is constant, then putting $F(x)=k(1), G(x)=a, H=h$ and $K(x)=-a h(x)+k(1)$, we have

$$
F(x y)=k(1)=G(x) H(y)+K(y), \quad x, y \in S,
$$

and, by (2.8),

$$
|K(x)-k(x)| \leq 2 \varepsilon, \quad x \in S .
$$

- If there are $x_{1}, x_{2} \in S$ with $g\left(x_{1}\right)=: a \neq b:=g\left(x_{2}\right)$ then, by (2.8) applied for $x_{1}$ and $x_{2}$ in place of $x$, we have

$$
|a h(y)+k(y)-k(1)| \leq 2 \varepsilon, \quad y \in S,
$$

and

$$
|b h(y)+k(y)-k(1)| \leq 2 \varepsilon, \quad y \in S,
$$

respectively. We conclude that

$$
|a-b||h(y)| \leq 4 \varepsilon, \quad y \in S,
$$

hence $h$ is bounded. Now, by (2.9), $k$ is also bounded. With $F=G=H=$ $K=0$ we get what is required.

3. $h(1) \neq 0$.

We put

$$
h_{1}(x):=\frac{h(x)}{h(1)}, k_{1}(x):=\frac{k(x)-k(1)}{h(1)}, \quad x \in S .
$$

After dividing both sides of (2.8) by $|h(1)|$ we get

$$
\left|g(x y)-g(x) h_{1}(y)-k_{1}(y)\right| \leq \frac{2 \varepsilon}{|h(1)|}=: \delta, \quad x, y \in S .
$$

3.1. $g$ and $k_{1}$ are bounded.

By (2.7) $f$ is also bounded, so it is enough to put $F=0, G=0$, $H=h$ and $K=0$ to get the assertion of the Theorem.

3.2. $g$ is bounded and $k_{1}$ is not.

By (2.7) $f$ is also bounded $\left(M_{1}:=\sup _{x \in S}|f(x)|\right)$, and by (2.10) we infer that $h_{1}$ is unbounded. Let $M_{2}>0$ be such that $|g(x)| \leq M_{2}$ for $x \in S$ and let $\left(y_{n}\right)_{n \in \mathbb{N}}$ be a sequence such that $0 \neq\left|h_{1}\left(y_{n}\right)\right| \stackrel{n \rightarrow \infty}{\longrightarrow} \infty$. By (2.10), for any $x_{1}, x_{2} \in S$, we obtain

$$
\left|g\left(x_{1}\right) h_{1}\left(y_{n}\right)+k_{1}\left(y_{n}\right)\right| \leq \delta+M_{2}, \quad n \in \mathbb{N},
$$


and

$$
\left|g\left(x_{2}\right) h_{1}\left(y_{n}\right)+k_{1}\left(y_{n}\right)\right| \leq \delta+M_{2}, \quad n \in \mathbb{N},
$$

SO

$$
\left|g\left(x_{1}\right)-g\left(x_{2}\right)\right|\left|h_{1}\left(y_{n}\right)\right| \leq 2 \delta+2 M_{2}, \quad n \in \mathbb{N} .
$$

Thereby, $g$ is constant $(g(x)=: a$ for $x \in S)$. We put $F=0, G=g$, $H=h$ and $K(x)=-a h(x), x \in S$. It is obvious that (2.2) is satisfied. In order to finish the proof in this case it is enough to check that the difference $k-K$ is bounded. By (2.1) we have

$$
|k(x)-K(x)|=|a h(x)+k(x)| \leq \varepsilon+M_{1}, \quad x \in S .
$$

3.3. $g$ is unbounded.

By Lemma 2.2 we infer that

$$
h_{1}(x y)=h_{1}(x) h_{1}(y), \quad x, y \in S .
$$

On account of Lemma 2.3 there exists $k_{2}: S \rightarrow \mathbb{C}$ such that

$$
k_{2}(x y)=k_{2}(x) h_{1}(y)+k_{2}(y), \quad x, y \in S,
$$

and

$$
\left|k_{2}(x)-k_{1}(x)\right| \leq 2 \delta, \quad x \in S
$$

We define

$$
\begin{aligned}
& F(x)=\left[k_{2}(x)+g(1) h_{1}(x)\right] h(1), \\
& G(x)=k_{2}(x)+g(1) h_{1}(x), \\
& H(x)=h_{1}(x) h(1)=h(x), \\
& K(x)=k_{2}(x) h(1), \quad x \in S .
\end{aligned}
$$

It easy to check, using (2.11) and (2.12), that (2.2) is fulfilled. Moreover, we have

$$
\begin{aligned}
|k(y)-K(y)| & =\left|k(y)-k_{2}(y) h(1)\right| \\
& \leq\left|k(y)-k_{1}(y) h(1)\right|+\left|k_{1}(y) h(1)-k_{2}(y) h(1)\right| \\
& \leq|k(1)|+2 \delta|h(1)|, \quad y \in S, \\
|g(x)-G(x)| & =\left|g(x)-k_{2}(x)-g(1) h_{1}(x)\right| \\
& \leq\left|g(x)-g(1) h_{1}(x)-k_{1}(x)\right|+\left|k_{1}(x)-k_{2}(x)\right| \\
& \leq \delta+2 \delta, \quad x \in S, \\
|f(x)-F(x)| & =|f(x)-G(x) h(1)| \\
\leq & |f(x)-g(x) h(1)-k(1)|+|k(1)|+|g(x)-G(x)||h(1)| \\
\leq \varepsilon & +|k(1)|+3 \delta|h(1)|, \quad x \in S .
\end{aligned}
$$




\section{Acknowledgements}

The research of the second author was supported by Institute of Mathematics, University of Silesia, Katowice, Poland (Stability of selected functional equations program). We gratefully acknowledge the Referee's suggestions to improve the style of the presentation.

Open Access. This article is distributed under the terms of the Creative Commons Attribution License which permits any use, distribution, and reproduction in any medium, provided the original author(s) and the source are credited.

\section{References}

[1] Aczél, J.: Vorlesungen über Funktionalgleichungen und ihre Anwendungen. Birkhäuser Verlag Basel, 1961. English edition: Lectures on Functional Equations and their Applications, Academic Press, New York (1966)

[2] Aczél, J.: On Applications and Theory of Functional Equations. Birkhäuser, Basel (1969)

[3] Aczél, J., Dhombres, J.: Functional Equations in Several Variables. Cambridge University Press, Cambridge (1989)

[4] Ebanks, B.: General solution of a simple Levi-Civitá functional equation on non-abelian groups. Aequationes Math. 85(3), 359-378 (2013)

[5] Járai, A., Székelyhidi, L.: Regularization and general methods in the theory of functional equations. Aequationes Math. 52(1), 10-29 (1996)

[6] McKiernan, M.A.: Equations of the form $H(x \circ y)=\sum_{i} f_{i}(x) g_{i}(y)$. Aequationes Math. 16(1-2), 51-58 (1977)

[7] Shulman, E.: Functional equations of homological type, Ph.D. Thesis, Moscow Institute of Electronics and Mathematics, Moscow 1994 (Russian)

[8] Shulman, E.: Group representations and stability of functional equations. J. London Math. Soc. 52(2), 111-120 (1996)

[9] Stetkaer, H.: Functional equations and matrix-valued spherical functions. Aequationes Math. 69(3), 271-292 (2005)

[10] Székelyhidi, L.: On the Levi-Civita functional equation. Ber. Math.-Statist. Sekt. Forsch. Graz, vol. 301, pp. 23 (1988)

[11] Székelyhidi, L.: Stability properties of functional equations describing the scientific laws. J. Math. Anal. Appl. 150, 151-158 (1990)

[12] Vincze, E.: Eine allgemeinere Methode in der Theorie der Funktionalgleichungen I. Publ. Math. Debrecen 9, 149-163 (1963)

Roman Badora and Barbara Przebieracz

Instytut Matematyki

Uniwersytet Śląski

Bankowa 14

40-007 Katowice

Poland

e-mail: robadora@math.us.edu.pl;

barbara.przebieracz@us.edu.pl 
Peter Volkmann

Institut für Analysis

KIT

76128 Karlsruhe

Germany

Received: February 23, 2014

Revised: July 10, 2014 